REZENSION

\section{Open Codes}

\section{machen noch keine offene Gesellschaft}

\author{
„Kollektivität und Macht im Internet“
}

\author{
Maximilian Roßmann, Institut für Technikfolgenabschätzung \\ und Systemanalyse (ITAS), Karlsruher Institut für Technologie (KIT), \\ Karlstraße 11, 76133 Karlsruhe (maximilian.rossmann@kit.edu)
}

Als Ulrich Dolata und Jan-Felix Schrape vom Institut für Soziologie der Universität Stuttgart 2013 das Diskussionspapier „Zwischen Individuum und Organisation: Neue kollektive Akteure und Handlungskonstellationen im Internet" als Forschungsskizze veröffentlichten, diagnostizierten die beiden Autoren einen Nachholbedarf der soziologischen Internetforschung: Zwar habe es zahlreiche Arbeiten zum Einfluss von Crowd, Swarms und Networks gegeben, doch mangele es an vergleichenden, konzeptionellen Arbeiten, die eine kritische Analyse dieser Phänomene in ihrem soziotechnischen Kontext ermöglichten. Mit der Veröffentlichung ihres Buches „Collectivity and Power on the Internet" bei SpringerBriefs liegen nun einschlägige, teilweise bereits publizierte Arbeiten von Dolata und Schrape gebündelt in einem englischsprachigen und einem deutschsprachigen Band vor.

Die beiden Autoren bieten unter dem Vorsatz organisationssoziologischer Anschlussfähigkeit eine detaillierte Synopse des Forschungsfeldes. Die Leitfrage des Buches wird dabei theoretisch, historisch und anhand ökonomischer Daten zu beantworten versucht: Wie ermöglichen, leiten, beeinflussen und verändern technische Infrastrukturen im Internet die Möglichkeiten der Selbstorganisation kollektiver Akteure und welche gesellschaftlichen Folgen lassen sich darin beobachten? Dolata und Schrape untersuchen am Leitfaden dieser und verwandter Fragen soziale Bewegungen, Open-Source-Projekte sowie die zunehmende Machtkonzentration einer Handvoll Internet-Giganten - und ihr auf empirische Analyse zielendes Konzept lässt ausreichende, kritische Distanz nicht vermissen.

This is an article distributed under the terms of the Creative Commons Attribution License CCBY 4.0 (https://creativecommons.org/licenses/by/4.0/)

https://doi.org/10.14512/tatup.27.2.74

\section{Soziale Bewegungen, Open Source und Internet-Godzillas}

Das Buch ist gegliedert in ein einleitendes Kapitel und vier exemplarische Bereiche und Kapitel, in denen Kollektivität und Macht spezifische Formen annehmen. In den ersten beiden Kapiteln entwickeln die Autoren das Analyseschema für Macht im Internet und deren Akteurstypen. Die Kapitel 4 und 5 beobachten und erklären Tendenzen der Machtzentrierung zu großen Plattformanbietern im Wechselverhältnis mit Open-Source-Projekten.

Dolata und Schrape folgen in ihrem analytischen Konzept einer Ausweitung der klassischen Differenz von Individuum und Organisation des akteurszentrierten Institutionalismus (Scharpf und Mayntz 1995): Nichtorganisierte Individuen können sich bei vergleichbarer Orientierung als Aggregat verhalten; kollektive Akteure verfügen über bedingte Möglichkeiten intentionalen Handelns ohne dabei wie korporative Akteure explizit bindende Hierarchie- und Mitgliedschaftsbestimmungen auszubilden (Dolata und Schrape 2018, S. 9 ff.). Diese Perspektive trifft im Buch auf die Beobachtung von Lawrence Lessigs „Code is Law“ (2006, S. 5): Technik sei ein potenziell funktionales Äquivalent institutioneller Restriktionen, wie exemplarisch an der algorithmischen News-Filterung im Vergleich zur journalistischen Selektionslogik erläutert wird (Dolata und Schrape 2018, S. 4149). Feedback-Systeme, Like-Buttons, Benutzeroberflächen und Twitter Emojis erzeugen gegenseitige Beobachtungs- und Koordinationsmöglichkeiten sozialer Aggregate, die sich bei zunehmender Qualität der Selbst-Referenz in Swarms, Crowds, Publics, Communities, Social Movements und Corporations unterscheiden lassen: Kollektive Akteure entstehen, wenn Kollektive beginnen, sich hinreichend reflexiv zum Bespiel auf die Bedeutung ihrer 100, 1.000 oder 10.000.000 Likes, Shares oder Follower zu beziehen und darüber eine stabilisierende Gruppenidentität, reflektierte Perspektive oder Zielorientierung ausbilden (ebd., S. 14, 41; vgl. auch Luhmann 1987, S. 601).

Im weiteren Verlauf differenzieren die Autoren auf Basis einschlägiger Studien weitere Akteursformen: Elitestrukturierte, klar fokussierte Gruppen wie Wikileaks oder PirateBay kön-

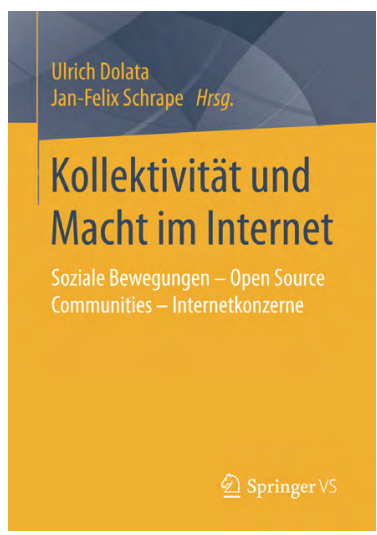

Dolata, Ulrich; Schrape, Jan-Felix (2018): Collectivity and power on the internet. A sociological perspective. Wiesbaden: SpringerBriefs in Sociology, 53,00 EUR, ISBN 978-3319784137

Dolata, Ulrich; Schrape, Jan-Felix (2018): Kollektivität und Macht im Internet Soziale Bewegungen - Open Source Communities - Internetkonzerne; Wiesbaden: Springer VS, 27,00 EUR, ISBN 978-3658179090 
nen sich beispielsweise auf Basis eigener technischer Onlineplattformen in geschlossenen Kernstrukturen auf qualitativ andere Weise auf subversive Aktivitäten abzielen, während hingegen klassische soziale Bewegungen, wie die G8/G20-Proteste, die TTIP/CETA-Proteste oder die lose vernetzte Occupy-Bewegung in Kommunikation, Koordination und Mobilisierung stark auf bestehende webbasierte Infrastrukturen zurückgreifen (ebd., S. 20f.): Während Kollektive einerseits von offenen Internetplattformen wie YouTube, WhatsApp oder Twitter sowie durch Wikis und E-Mailverteiler profitieren (ebd., S. 34, 40), unterliegen sie in ihrer Autonomie andererseits den Algorithmen der Plattformanbieter und sind anfällig für willkürliche Restriktionen, Zensur, Löschung und Überwachung durch Unternehmen, Geheimdienste oder autoritäre Regierungen (ebd., S. 42 f.). Zwar wird das Internet zum wichtigsten Ausgangspunkt neuer sozialer Formationen (ebd., S. 22), doch mit dem Hinweis auf die identitätsbildende und koordinierende Funktion charismatischer Anführer, geteilter Weltbilder, Visionen, Face-to-face-Kommunikation und ,klassischer" Leitmedien wie dem Fernsehen, positionieren sich die Autoren explizit gegen die Annahme einer Unabhängigkeit des Digitalen (ebd., S. 49 ff.). Diesem Wechselspiel kollektiver Akteure und ihrer ermöglichenden und ermöglichten Online-Plattformen folgen auch die weiteren Kapitel über Open Source und die Machtverschiebung hin zu den Internetgiganten.

Open-Source-Communities sind durch den rebellischen Charakter der anfänglichen Gründungsutopien verwandt mit offenen Bewegungen und stehen in einem Spannungsverhältnis zu klassischen Organisationsformen: Unabhängigkeit von Marktzwängen, Regeln, Lizenzen und Hierarchien sollten der Freiheit und Kreativität Vorschub leisten (ebd., S. 58). Die idealtypisch-differenzierte Analyse von 21 Open-Source-Projekten zeichnet ein anderes Bild (ebd., 68 ff.): Die bekanntesten Projekte, wie zum Beispiel der Linux Kernel, Ubuntu, Wordpress und Mozilla Firefox sind hierarchisch organisiert. Zudem haben externe Unternehmen Einfluss, indem sie einerseits Entwickler zu Verfügung stellen oder über Stiftungen Leitungspositionen besetzen, wie zum Beispiel bei Android oder dem Cloudservice OpenStack. Auch heterarchische Infrastrukturprojekte, wie zum Beispiel Typo3 und OpenSSL, sind auf Firmenspenden und explizite Repräsentationspositionen angewiesen. Nur wenige und kleinere Open-Source-Projekte bleiben dem ursprünglichen Narrativ treu und verzichten auf formale Entscheidungsstrukturen, Hierarchien sowie den größeren Einfluss und die finanzielle Abhängigkeit von großen Unternehmen. Die OpenSource-Praxis lässt sich deshalb heute vor allem als wichtige Innovationsstrategie einer Branche mit schnellen Innovationszyklen beschreiben - doch offene Quellcodes machen noch keine offene Gesellschaft (ebd., S. 76 ff.).

Aus anderer Perspektive, die einer empirischen Widerlegung digitaler Befreiungsutopien gleichkommt, beobachtet und erklärt Dolata die zunehmender Machtkonzentration auf wenige Organisationen (ebd., S. 85): Unternehmen wie Amazon, Apple, Facebook, Google und Microsoft festigen ihren Erfolg als Plattformanbieter durch Netzwerkeffekte. Sie können Einfluss neh- men auf das Nutzerverhalten zum Beispiel über Datenprofile und Algorithmen. Innerhalb eigener "Ökosysteme“ nehmen sie Einfluss auf Markt und Wettbewerb durch Gestaltung von App-Stores-Regeln, Entwicklertools und Hardwareschnittstellen (ebd., S. 91, 101). Während die eigenen Forschungs- und Entwicklungsabteilungen vor allem vorhandene Technologien weiterentwickeln, erschließen die großen Unternehmen neue Bereiche vor allem durch Aufkaufen kleinerer Startups. Die Mächtigen pflegen und nutzen die Nähe zu Open-Source-Projekten als strategisches Innovationsfeld, profitieren durch das Ökosystem ihrer Plattformen von Erfolgen, übernehmen bewährte Praktiken oder kaufen schlicht ihre Betriebe auf (ebd., S. 93 ff.).

\section{Fazit}

Zusammengefasst beobachten die Autoren eine Machtkonzentration im Internet auf wenige Akteure, doch enden sie schließlich mit dem Hinweis, dass verändertes Nutzerverhalten und politische Regularien diese Machtverhältnisse weiterhin in Frage stellen können (ebd., S. 105) - und sollten? Das Buch liest sich einerseits als gelungene Erklärung von Macht und Kollektivität im Internet entlang der Co-Evolution von Open-Source-Projekten und Plattformanbietern. Es mag durchaus überraschen, dass all die großen Open-Source-Projekte klassische Organisationsformen teilen und in ökonomischer Abhängigkeit zu den Internet-Riesen existieren. Ein Vergleich mit ähnlichen Symbiosen im ostasiatischen Raum, wie Alibaba, Baidu, Tencent und Xiaomi (mit eigenem Android UI) blieb dabei leider aus. Andererseits taugt das Buch auch als praktische Einführung in die problemorientierte Forschungsheuristik des akteurszentrierten Institutionalismus. Hervorzuheben ist die Kritik am Technik-Determinismus der Digital-Utopien, deren Funktion bereits an anderer Stelle in Co-Autorenschaft mit Sascha Dickel (2017) aufgegriffen wurde: Utopische Kommunikation in der digitalen Welt dient gerade nicht der Zukunftsprognose, sondern als „gegenwärtige Zukunft“ einer sachlich-zeitlichen Orientierung und Mitgestaltung gegenwärtiger Diskurse und Selbstvergewisserung kollektiver Akteure. Die Ausgangspunkte und die Typologie des Buches zeigen sich jedenfalls äußerst vielversprechend für eine zeitgemäße und problemorientierte Technikgeneseforschung, die Bedingungen digitaler Welten ernst nimmt ohne davon geblendet zu sein.

\footnotetext{
Literatur

Dickel, Sascha; Schrape, Jan-Felix (2017): The logic of digital utopianism; In: NanoEthics 11 (1), S. 47-58

Dolata, Ulrich; Schrape, Jan-Felix (11/2013): Zwischen Individuum und Orga-

nisation. Neue kollektive Akteure und Handlungskonstellationen im

Internet. Stuttgart: Stuttgarter Beiträge zur Organisations- und Innovations-

forschung (2).

Lessig, Lawrence (2006): Code - Version 2.0; [2. ed.]; New York: Basic Books.

Luhmann, Niklas (1987): Soziale Systeme; 15. Aufl.; Frankfurt a. M.: Suhrkamp.

Mayntz, Renate; Scharpf, Fritz W. (1995) (Hrsg.): Gesellschaftliche Selbstregelung und politische Steuerung. In: Schriften des Max-Planck-Instituts für Gesellschaftsforschung Köln (23). Frankfurt a. M.: Campus.
} 